\title{
Response Surface Modeling of 100nm CMOS Process Technology using Design of Experiment
}

\author{
H. C. Srinivasaiah and Navakanta Bhat \\ Microelectronics Lab., Department of ECE, Indian Institute of Science, Bangalore-560012, India \\ E-mail:srinivas@protocol.ece.iisc.ernet.in ornavakant@ece.iisc.ernet.in
}

\begin{abstract}
$100 \mathrm{~nm}$ CMOS technology has been characterized through Design of Experiment (DOE) and statistical modeling. Initially, a set of 21 process parameters (factors) have been identified to determine their impact on transistor performance metrics such as threshold voltage $V_{t}$, subthreshold slope SS, drive current $I_{\text {ddrive, }}$, leakage current $I_{\text {dleak }}$, both in saturation and linear region. Through first order linear modeling of $V_{t}, S S, I_{d d r i v e}$, and $I_{\text {dleak }}$, a subset of 10 most significant process parameters are picked using Plackett-Burman screening experiment for both NMOS and PMOS devices. Significant process parameters which impact the device characteristics are seen to be different, for NMOS and PMOS devices, inspite of a common process flow. Response surfaces (RS) have been built in terms of these 10 parameters for NMOS device. Statistical parameters of the device characteristics fluctuations like mean $(\mu)$ and standard deviation $(\sigma)$ for $V_{t}$, SS, $I_{\text {ddrive }}, I_{\text {dleak }}$ and $G_{m}$ (maximum transconductance), have been determined by Monte carlo $(M C)$ analysis of these response surfaces. Application of the Transmission of Moment Technique (TMT) on these models is shown to be a simple means to determine $\mu$ and $\sigma$ of the device characteristics, with simple mathematical calculations.

Keywords - Disposable spacer, Sensitivity analysis, CMOS technology, Transmission of moments technique, Statistical modeling, PlackettBurman design, Response surface methodology, Monte carlo analysis.
\end{abstract}

\section{Introduction}

The transistor mismatch due to random variations in process parameters has become one of the major issue in the DSM technology. The term transistor mismatch refers to the fact that supposedly identical transistors at the design phase come out as distinct devices after manufacturing [1] due to statistical process variations. The process variations influence the transistor output parameters which in turn have a significant impact on circuit performance and yield [2-4]. The transistor mismatch effect will become worse in the future due to demanding requirement on process tolerance. The International Technology Roadmap for
Semiconductors (ITRS) expects to reduce the overall cost of manufacturing of the Deep Sub-Micron (DSM) ICs by $35 \%$ in the future, by the use of TCAD-tools [5]. The manufacturing yield of a technology is a strong function of statistical process variations. In order to decrease the Short Channel Effect (SCE), in DSM devices, additional process steps are involved. This increases the complexity in device design and process control. The statistical distribution of transistor parameters can be evaluated, in principle, through rigorous Monte Carlo analysis by accounting for the random statistical distribution of parameters of various processes such as lithography, oxidation, implantation etc. However this technique demands a lot of computing resources [6]. Hence it becomes essential to come up with a computationally efficient modeling technique for characterization.

In this work, we have initially explored a 21 dimensional parameter space to investigate the relative significance of each parameter for $100 \mathrm{~nm}$ CMOS technology. Next, we have considered top 10 significant parameters that most influence the device behavior. Finally, second order response surfaces for NMOS device characteristics have been determined in terms of the corresponding 10 significant process parameters, which will enable the process engineering and manufacturing team to track the process and monitor for the desired target and minimum variability. In Section II, we discuss the principles of statistical modeling, in section III, design of 100nm NMOS and PMOS. Section IV describes the process characterization for $100 \mathrm{~nm}$ CMOS using screening DOE technique. In section $\mathrm{V}$, a detailed response surface study is performed in terms of the 10 key process parameters and MC and TMT are applied on these response surfaces to evaluate the mean and total variability of responses. In Section VI, we conclude with the discussion on, the techniques used and the results that are obtained.

\section{Modeling Methodology}

Plackett-Burman DOE [7,8] with 28 runs for a maximum of 27 variables have been performed as screening experiment for the initial study of the CMOS process. A first 
order model for a response y, with 21 variables is of the form [8]

$\mathrm{y}=\mathrm{b}_{0}+b_{1} x_{1}+b_{2} x_{2}+\ldots+b_{21} x_{21}$

where $b_{0}$ is a constant, $x_{i} \mathrm{~s}$ are the normalized process parameters varying between -1 to +1 , and $b_{i}$ s are the corresponding fitting constants determined by the data obtained from the screening experiment, for $\mathrm{i}=1 \ldots 21$, as shown in Table 1 . The magnitude of $b_{i}$ in equation 1 indicates the relative significance of the corresponding $x_{i}$, as these $x_{i}$ s are normalized between -1 to +1 , with respect to their $3 \sigma$.

As a case study, second order response surfaces $[8,9]$ have been built using DOE, for NMOS device in terms of the 10 significant parameters. Remaining 11 parameters are set at their nominal values. The models fitted to data from a suitable DOE, contain a constant term, main factor terms, all possible two factor interaction terms and quadratic terms. Thus any response represented as a second order model will have a total of 66 terms for 10 factors, which will be of the form

$$
\begin{gathered}
\mathrm{y}=\beta_{0}+\beta_{1} x_{1}+\beta_{2} x_{2}+\ldots+\beta_{10} x_{10}+\beta_{12} x_{1} x_{2}+ \\
\beta_{13} x_{1} x_{3}+\ldots+\beta_{23} x_{2} x_{3}+\beta_{24} x_{2} x_{4}+\ldots+\beta_{910} x_{9} x_{10}+ \\
\beta_{11} x_{1}^{2}+\beta_{22} x_{2}^{2}+\ldots+\beta_{1010} x_{10}^{2}
\end{gathered}
$$

where $\beta_{0}$ is a constant, $x_{i} \mathrm{~s}$ are the most significant factors as determined from screening experiment, varying between -1 to +1 , and $\beta_{i}$ s are the corresponding fitting constants determined by the data obtained from the response surface DOE, for $\mathrm{i}=1 \ldots 10$. In general, response y can be any of the device characteristics, $V_{t}, \mathrm{SS}, I_{d \text { drive }}, I_{\text {dleak }}$ and $G_{m}$, in either linear or saturation region. Equation 2 can now be used in MC loop to determine the mean and variance of any response with 10 factors, which are assumed to be normal random variables. When the RS modeling is performed, TMT $[6,10]$ on these models, can readily obtain $\mu$ and $\sigma$ of the device parameters. According to TMT, any nonlinear relation $y=f\left(x_{1}, x_{2}, \ldots x_{N}\right)$ between a dependent response variable y and independent variables $x_{1}, x_{2}, \ldots x_{N}$ can be approximated as a truncated Taylor's series.

$$
y=f\left(\mu_{x_{1}}, \mu_{x_{2}}, \ldots \mu_{x_{N}}\right)+\sum_{i=1}^{N} \frac{\partial f}{\partial x_{i}} \Delta x_{i}
$$

where $\mu_{x_{i}}$ is the mean value of the $i^{\text {th }}$ process parameter. According to the TMT, mean of the response, $y$ is

$$
\mu_{y}=f\left(\mu_{x_{1}}, \mu_{x_{2}}, \ldots \mu_{x_{N}}\right)
$$

and the variance of $y$ is,

$$
\begin{aligned}
\sigma_{y}^{2}= & \sum_{i=1}^{N}\left(\frac{\partial f}{\partial x_{i}}\right)^{2} \sigma_{x_{i}}^{2}+2 \sum_{i=1}^{N} \sum_{j=i+1}^{N} \frac{\partial f}{\partial x_{i}} \frac{\partial f}{\partial x_{j}} \rho_{x_{i} x_{j}} \\
& \times \sigma_{x_{i}} \sigma_{x_{j}}
\end{aligned}
$$

where $\sigma_{x_{i}}^{2}$ is the variance of $x_{i}$ and $\rho_{x_{i} x_{j}}$ is the correlation between the variable $x_{i}$ and $x_{j}$. In equation 3 and 5 , partial derivatives are taken about the nominal point of the process parameters. If $x_{i} \mathrm{~s}$ are statistically independent then the second term in equation 5 , vanishes.

\section{Design of nominal NMOS and PMOS device}

We have used Integrated System EngineeringTechnology Computer Aided Design (ISE-TCAD) tool in the implementation of this work [11]. In realizing the nominal $0.1 \mu \mathrm{m}$ NMOS and PMOS (without silicidation) device of Figure 1, we have followed the technique outlined in [12-15]. A detailed process flow followed in DIOS-ISE process simulation which accounts for the initial 21 parameters is given in Table 1. The definition of the gate length appears at the top of the simulation command file, as it appears first, in Table 1. The shallow extension/deep junction depth for NMOS and PMOS transistor are $0.04 \mu \mathrm{m} / 0.1 \mu \mathrm{m}$ and $0.05 \mu \mathrm{m} / 0.15 \mu \mathrm{m}$ respectively. The gate source/drain ( $\mathrm{s} / \mathrm{d})$ overlap is maintained at about $15 \mathrm{~nm}$ for the parasitic series resistance considerations. The gate length $L_{g}=0.1 \mu \mathrm{m}$ (metallurgic channel length, $L_{m e t}=0.07 \mu \mathrm{m}$ ), oxide thickness $T_{o x}=20 \AA$ and these devices has been simulated at a supply voltage, $\left|V_{d s}\right|=1.5 \mathrm{~V}$. Figure 2 shows the $I_{d}-V_{g s}$ characteristics simulated in the saturation $\left(\left|V_{d s}\right|=1.5 \mathrm{~V}\right)$ and linear $\left(\left|V_{d s}\right|=50 \mathrm{mV}\right)$ region for the NMOS and PMOS devices. DESSIS-ISE has been used for device simulation. An interface trap density of $5 \times 10^{10} / \mathrm{cm}^{2}$ is applied at the $\mathrm{Si} /$ Oxide boundary during the simulation. For NMOS the saturation/linear region, $V_{t}=0.233 / 0.31 \mathrm{~V}, S S=85.8 / 83.6 \mathrm{mV} / \mathrm{dec}$, $I_{\text {ddrive }}=0.91 / 0.124 \mathrm{~mA} / \mu \mathrm{m}, I_{\text {dleak }}=0.69 / 0.064 \mathrm{nA} / \mu \mathrm{m}$ and $G_{m}=0.92 / 0.14 \mathrm{mS} / \mu \mathrm{m}$. The respective quantities for PMOS are $0.23 / 0.26 \mathrm{~V}, \quad 84.1 / 84.0 \mathrm{mV} / \mathrm{dec}, \quad 0.33 / 0.034 \mathrm{~mA} / \mu \mathrm{m}$, $0.93 / 0.29 \mathrm{nA} / \mu \mathrm{m}$ and $0.35 / 0.04 \mathrm{mS} / \mu \mathrm{m}$ respectively. The $V_{t} \mathrm{~s}$ that are indicated above are the constant current $V_{t}$ (@) $40 n A \times\left(W / L_{g}\right)$, with $W=1 \mu \mathrm{m}$ and $\left.L_{g}=0.1 \mu \mathrm{m}\right)$.

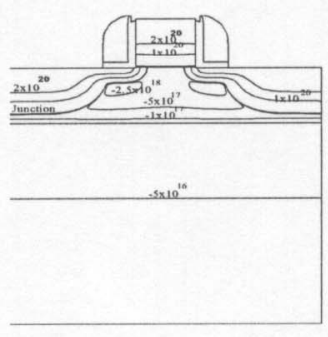

(a)

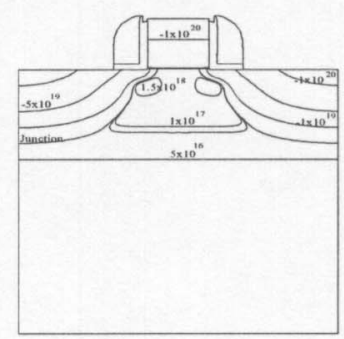

(b)
Figure 1. Process simulated (a)NMOS and (b)PMOS devices, contours of doping concentration can be seen 
Table 1. Symbolic representation of the 21 process parameters, respective model coefficients in equation $\mathbf{1}$, their nominal and $3 \sigma$ values. Values in the parentheses pertain to $\mathrm{PMOS}$. coeff=model coefficient, SSRC=super steep retrograde channel, LDD=light doped drain, RTA=rapid thermal anneal.

\begin{tabular}{|c|c|c|c|}
\hline factors & coeff & factor description & $3 \sigma$ \\
\hline$\overline{\mathrm{Lg}}$ & $\overline{\mathrm{b}_{1}}$ & gate length $=0.1(0.1) \mu \mathrm{m}$ & $0.01(0.01) \mu \mathrm{m}$ \\
\hline initoxtemp & $\mathrm{b}_{2}$ & 10nm screening oxide@ $@ \mathrm{~T}=900(900)^{\circ} \mathrm{C}$ & $10(10)^{\circ} \mathrm{C}$ \\
\hline ztiltdose & $\mathrm{b}_{3}$ & $0^{\circ}$ implant $\mathrm{B}(\mathrm{P})$ dose $=2(2) \times 10^{12} / \mathrm{cm}^{2}$ & $0.2(0.2) \times 10^{12} / \mathrm{cm}^{2}$ \\
\hline ztiltenergy & $\mathrm{b}_{4}$ & $0^{\circ}$ implant energy $=200(400) \mathrm{keV}$ & $20(40) \mathrm{keV}$ \\
\hline tiltdose & $b_{5}$ & $7^{\circ}$ implant B (P) dose $=1(2) \times 10^{12} / \mathrm{cm}^{2}$ & $0.1(0.2) \times 10^{12} / \mathrm{cm}^{2}$ \\
\hline tiltenergy & $\mathrm{b}_{6}$ & $7^{\circ}$ implant energy $=100(200) \mathrm{keV}$ & $10(20) \mathrm{keV}$ \\
\hline Ssrc_dose & $\mathrm{b}_{7}$ & In(Sb) SSRC dose $=5.7(5.7) \times 10^{12} / \mathrm{cm}^{2}$ & $0.57(0.57) \times 10^{12} / \mathrm{cm}^{2}$ \\
\hline ssrc_energy & $b_{8}$ & In(Sb) SSRC energy $=146(186) \mathrm{keV}$ & $14.6(18.6) \mathrm{keV}$ \\
\hline goxtemp & $\mathrm{b}_{9}$ & gate oxidation@ $\mathrm{T}=800(800)^{\circ} \mathrm{C}$ & $10(10)^{\circ} \mathrm{C}$ \\
\hline reoxtemp & $\mathrm{b}_{10}$ & poly-reoxidation@ @ $\mathrm{T}=800(800)^{\circ} \mathrm{C}$ & $10(10)^{\circ} \mathrm{C}$ \\
\hline spnithick1 & $\mathrm{b}_{11}$ & spacer thickness $=96(96) \mathrm{nm}$ & $9.6(9.6) \mathrm{nm}$ \\
\hline deep_dose & $\mathrm{b}_{12}$ & deep s/d As(B) dose $=6(1) \times 10^{15} / \mathrm{cm}^{2}$ & $0.6(0.1) \times 10^{15} / \mathrm{cm}^{2}$ \\
\hline deep_energy & $\mathrm{b}_{13}$ & deep $\mathrm{s} / \mathrm{d}$ energy $=30(5) \mathrm{keV}$ & $3(0.5) \mathrm{keV}$ \\
\hline anneal_templ & $b_{14}$ & 20s anneal@T=1050(1050) ${ }^{\circ} \mathrm{C}$ & $10(10)^{\circ} \mathrm{C}$ \\
\hline ldd_dose & $\mathrm{b}_{15}$ & LDD As(B) dose $=1(0.1) \times 10^{15} / \mathrm{cm}^{2}$ & $0.1(0.01) \times 10^{15} / \mathrm{cm}^{2}$ \\
\hline ldd_energy & $\mathrm{b}_{16}$ & LDD energy $=7(1) \mathrm{keV}$ & $0.7(0.1) \mathrm{keV}$ \\
\hline halo_dose & $\mathrm{b}_{17}$ & halo $\mathrm{B}(\mathrm{P})$ dose $=6.65(5.85) \times 10^{12} / \mathrm{cm}^{2}$ & $0.665(0.585) \times 10^{12} / \mathrm{cm}^{2}$ \\
\hline halo_energy & $\mathrm{b}_{18}$ & halo energy $=10(25) \mathrm{keV}$ & $1(2.5) \mathrm{keV}$ \\
\hline halo_tilt & $\mathrm{b}_{19}$ & halo tilt $=30(30)^{\circ}$ & $3(3)^{\circ}$ \\
\hline anneal_temp2 & $\mathrm{b}_{20}$ & 4s anneal@T=1050 $(1050)^{\circ} \mathrm{C}$ & $10(10)^{\circ} \mathrm{C}$ \\
\hline spnithick2 & $\mathrm{b}_{21}$ & final spacer thickness $=40(40) \mathrm{nm}$ & $4(4) \mathrm{nm}$ \\
\hline
\end{tabular}

Table 2. Coefficients of first order model for NMOS (PMOS).

\begin{tabular}{|c|c|c|c|c|}
\hline coeff & $\begin{array}{c}V_{\text {tsat }} \\
\times 10^{-3}\end{array}$ & $S S_{\text {sat }}$ & $\begin{array}{c}I_{\text {dsatdrive }} \\
\times 10^{-6}\end{array}$ & $\begin{array}{c}I_{\text {dsatleak }} \\
\times 10^{-9}\end{array}$ \\
\hline \hline $\mathrm{b}_{0}$ & $220(180)$ & $87.6(82.4)$ & $940(420)$ & $5.4(15.5)$ \\
\hline $\mathrm{b}_{1}$ & $24(2.4)$ & $-3.24(-0.9)$ & $-110(-13)$ & $-4.5(-7.9)$ \\
\hline $\mathrm{b}_{2}$ & $8.2(-0.12)$ & $-0.19(-0.52)$ & $12.9(6)$ & $-2.7(2.6)$ \\
\hline $\mathrm{b}_{3}$ & $1.5(-0.63)$ & $0.05(-0.52)$ & $-5(3)$ & $2(5.6)$ \\
\hline $\mathrm{b}_{4}$ & $-1.3(-5.1)$ & $-0.09(-0.19)$ & $13.5(2.4)$ & $-1.1(1.8)$ \\
\hline $\mathrm{b}_{5}$ & $1(-1.3)$ & $0.12(-0.3)$ & $-15.8(1.2)$ & $-2.1(-4)$ \\
\hline $\mathrm{b}_{6}$ & $0.14(-4.1)$ & $-0.22(-0.69)$ & $13(4.6)$ & $2.3(1.5)$ \\
\hline $\mathrm{b}_{7}$ & $7(8)$ & $-0.24(-0.19)$ & $-7.6(-8.7)$ & $-2.2(-7.9)$ \\
\hline $\mathrm{b}_{8}$ & $-24(-10)$ & $0.88(1.42)$ & $2(5)$ & $3.6(-2.5)$ \\
\hline $\mathrm{b}_{9}$ & $10(7)$ & $0.61(0.62)$ & $8.3(-5.3)$ & $-1.2(2.8)$ \\
\hline $\mathrm{b}_{10}$ & $7(9.5)$ & $0.6(0.52)$ & $-53.6(-14)$ & $1.9(-1.1)$ \\
\hline $\mathrm{b}_{11}$ & $0.72(35)$ & $0.22(0.86)$ & $-22(-39)$ & $-2.1(-13.6)$ \\
\hline $\mathrm{b}_{12}$ & $-2.6(-21)$ & $-0.26(-0.87)$ & $23.6(22)$ & $1.6(9.5)$ \\
\hline $\mathrm{b}_{13}$ & $-1.2(-1.4)$ & $-0.08(0.05)$ & $19.9(2.2)$ & $1.5(1.9)$ \\
\hline $\mathrm{b}_{14}$ & $-2.6(-23)$ & $-0.48(-0.98)$ & $47.1(24)$ & $1.9(12.4)$ \\
\hline $\mathrm{b}_{15}$ & $-8.7(-13)$ & $0.3(0.75)$ & $16.9(14)$ & $2.7(7.8)$ \\
\hline $\mathrm{b}_{16}$ & $-4.8(-4.2)$ & $0.31(0.59)$ & $5.2(7.4)$ & $3.1(-0.02)$ \\
\hline $\mathrm{b}_{17}$ & $21(22)$ & $-0.1(0.23)$ & $-24.7(-18)$ & $-2.7(-4.4)$ \\
\hline $\mathrm{b}_{18}$ & $16(9.5)$ & $-0.56(-0.54)$ & $-29.5(-6.8)$ & $-3.5(-3.4)$ \\
\hline $\mathrm{b}_{19}$ & $28(28)$ & $-0.62(-0.03)$ & $-38.7(-17)$ & $-3.9(-6.7)$ \\
\hline $\mathrm{b}_{20}$ & $-25(-14)$ & $1.23(0.49)$ & $38.1(14)$ & $3.7(10)$ \\
\hline $\mathrm{b}_{21}$ & $-1.4(-5.6)$ & $0.17(-0.1)$ & $-7.2(4)$ & $-1(4.7)$ \\
\hline & & & & \\
\hline
\end{tabular}

Table 3. Top 10 statistically significant process parameters for NMOS and PMOS.

\begin{tabular}{|l|l|l|}
\hline Rank & NMOS & PMOS \\
\hline \hline 1 & Lg & spnithick1 \\
\hline 2 & halo_tilt & anneal_temp1 \\
\hline 3 & anneal_temp2 & deep_dose \\
\hline 4 & ssrc_energy & halo_tilt \\
\hline 5 & halo_energy & anneal_temp2 \\
\hline 6 & halo_dose & ldd_dose \\
\hline 7 & reoxtemp & halo_dose \\
\hline 8 & ldd_dose & Lg \\
\hline 9 & Initoxtemp & ssrc_dose \\
\hline 10 & anneal_temp1 & ztiltdose \\
\hline
\end{tabular}

Table 4. Statistics that are obtained by MC and TMT calculations on the response surfaces.

\begin{tabular}{|l|l|l|l|c|}
\hline \multirow{2}{*}{$\begin{array}{l}\text { Device } \\
\text { parameters }\end{array}$} & \multicolumn{2}{|c|}{ MC } & \multicolumn{2}{c|}{ TMT } \\
\cline { 2 - 5 } & Mean & $\sigma$ & Mean & $\sigma$ \\
\hline \hline$V_{\text {tsat }}(\mathrm{V})$ & 0.243 & 0.019 & 0.246 & 0.018 \\
\hline$V_{\text {tlin }}(\mathrm{V})$ & 0.316 & 0.014 & 0.319 & 0.014 \\
\hline$S S_{\text {sat }}(\mathrm{mV} / \mathrm{dec})$ & 87.06 & 1.367 & 87.0 & 1.33 \\
\hline$S S_{\text {lin }}(\mathrm{mV} / \mathrm{dec})$ & 84.64 & 0.949 & 84.7 & 0.93 \\
\hline$I_{\text {dsatdrive }}(\mu \mathrm{A} / \mu \mathrm{m})$ & 832 & 58.4 & 820 & 53.4 \\
\hline $\log \left[I_{\text {dsatleak }} \mathrm{A} / \mu \mathrm{m}\right]$ & -21.2 & 0.41 & -21.3 & 0.4 \\
\hline$I_{\text {dlindrive }}(\mu \mathrm{A} / \mu \mathrm{m})$ & 113 & 5.1 & 112 & 4.1 \\
\hline $\log \left[I_{\text {dlinleak }} \mathrm{A} / \mu \mathrm{m}\right]$ & -23.6 & 0.394 & -23.65 & 0.38 \\
\hline$G_{\text {msat }}(\mathrm{mS} / \mu \mathrm{m})$ & 0.869 & 0.05 & 0.86 & 0.047 \\
\hline$G_{\text {mlin }}(\mathrm{mS} / \mu \mathrm{m})$ & 0.138 & 0.007 & 0.14 & 0.005 \\
\hline
\end{tabular}




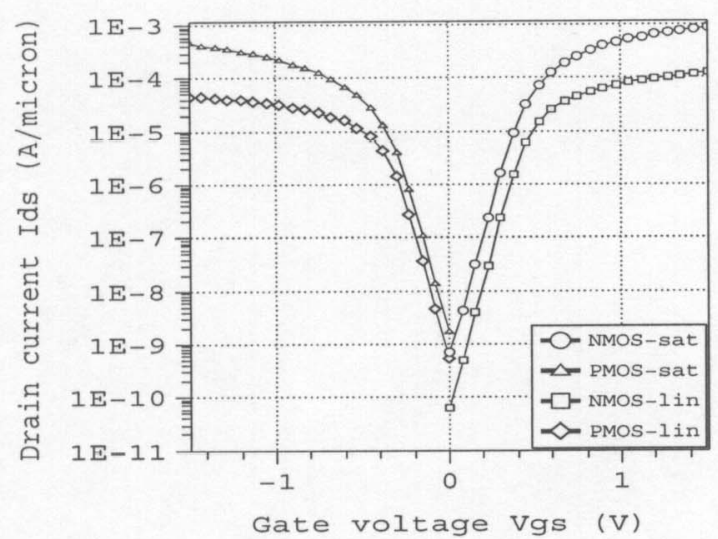

Figure 2. $I_{d}-V_{g s}$ characteristics of NMOS and PMOS devices in the linear and saturation region

\section{Screening experiment for characterizing 100nm CMOS process}

Plackett-Burman DOE with 28 runs for a maximum of 27 variables, have been performed as screening experiment for the initial study of the CMOS process. The number of process parameters that are investigated by this DOE are 21 , which are tabulated in Table 1 with their description (quantities in the parentheses, pertains to PMOS). The standard design matrix for Plackett-Burman experiment with a maximum of 27 variables with 28 runs can be found in $[7,8]$. The experiment has been performed by assigning 21 process parameters to each column of the standard design matrix (omitting the last 6 columns). The whole experiment has been repeated 4 times by changing the process parameter allocation to columns of the design matrix. Model coefficients of equation 1 are determined by single regression of the results from all the 4 experiments. This is essential only to increase the effectiveness of the screening experiment in properly deciding the significance (in terms of rank) of the 21 parameters under study. The first order model coefficient in equation 1 for $V_{t}, \mathrm{SS}, I_{d d r i v e}$ and $I_{\text {dleak }}$ in saturation region, obtained from this DOE are listed in Table 2 for NMOS and PMOS (in parentheses). Note that the subscript, $d=d r a i n$, sat=saturation, lin=linear, drive $=$ on-state and leak=leakage, in the further discussion. e.g $V_{\text {tsat }}=V_{t}$ in the saturation region, $I_{\text {dsatdrive }}=$ on-state drain current in the saturation region, etc. Equation 1 should not be used for extensive study except for determining the significance of the factors, because it is taking only the approximate first order effect of the process parameters. For a detail study of the process, with respect to these factors, one has to use response surface DOE and modeling, which will be discussed in the next section. The magnitude of the model coefficients will indicate the relative significance of the corresponding process parameter. This procedure helps in picking a subset of significant factors out of initial 21. Table 3 is the list of top 10 ranking factors for NMOS and PMOS device. It can be seen that this subset is not common between NMOS and PMOS devices, as the boron and arsenic diffusivity is different in silicon, even though the process flow is common, which have the direct impact on the SCE in the devices.

\section{Response surface modeling, Monte carlo and Transmission of moments analysis}

The response surface modeling, has been performed by using Face Centered Central Composite (FCCC) Design $[8,9,16]$ on the 10 , key process parameters of Table 3 , for NMOS device. This design is a highly fractionated 3 level DOE for fitting second order response surfaces. For 10 factors, a fraction of 1045 experiments have been chosen, out of a large set of $3^{10}(=59049)$ full factorial design. The process parameters under consideration are varied by $\pm 10 \%$ except temperatures, which are varied by $\pm 10^{\circ} \mathrm{C}$, about their nominal values. It is assumed that $\pm 10 \%$ or $\pm 10^{\circ} \mathrm{C}$, corresponds to $\pm 3 \sigma$ variation in the process under study. A total of 1045 process and 1045 device simulations have been performed. The tool flowchart that has been used in GENESISe framework is shown in Figure 3.

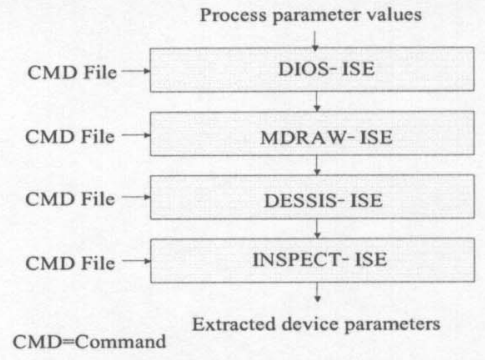

Figure 3. GENESISe tool flow chart for statistical modeling

GENESISe, [11] also called the work bench of ISETCAD tool is used to construct project and run, automatically. DIOS-ISE has been used to process simulate the devices and DESSIS-ISE, for device simulation. A boundary/doping editor tool MDRAW-ISE has been used to build mesh automatically. INSPECT-ISE has been used for automatic extraction of the simulation results. The second order models that have been obtained by the regression technique using simulation results are long polynomials, in the form of equation 2. The device parameters that have been modeled are $V_{t}, \mathrm{SS}, I_{\text {ddrive }}, I_{\text {dleak }}$ and $G_{m}$, in the saturation and linear region. These models have been tested for their validity to predict the response values, by substituting the same combination of the factor levels that have been used in the response surface DOE. The original experimental response and the model predicted response correlate very well with the correlation coefficient, $r>0.9$. 


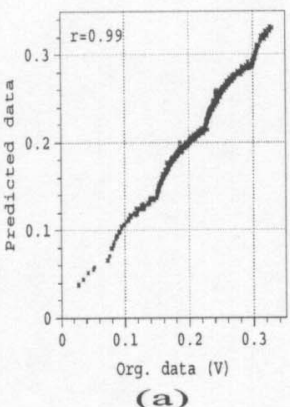

(a)

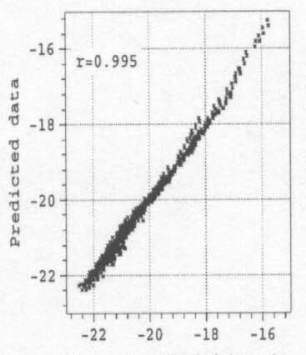

Org. data $\{\log [A /$ micron $]\}$

(f)
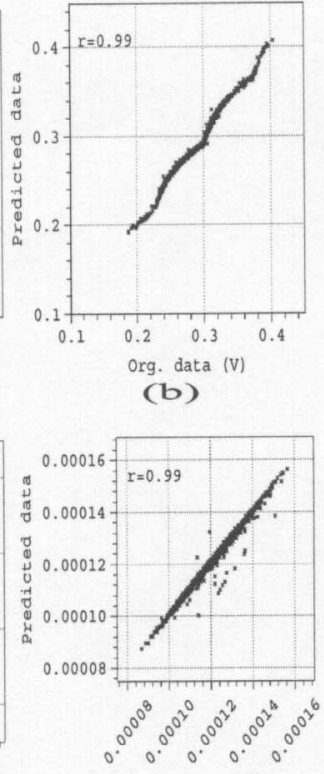

Org. data $(A)$

(g)
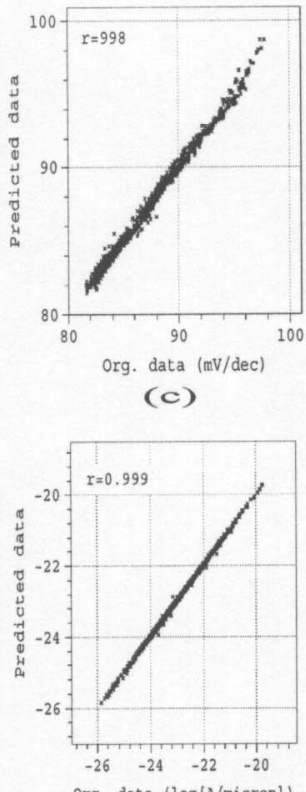

org. data $(\log [\mathrm{A} /$ micron $)$

(h)

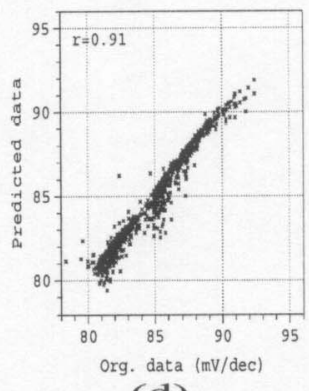

(d)

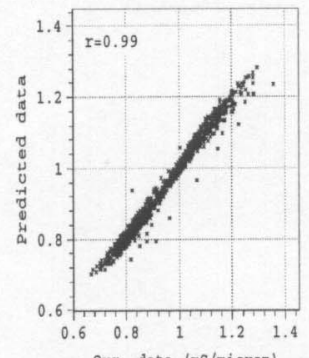

org. data (mS/micron)

(i)
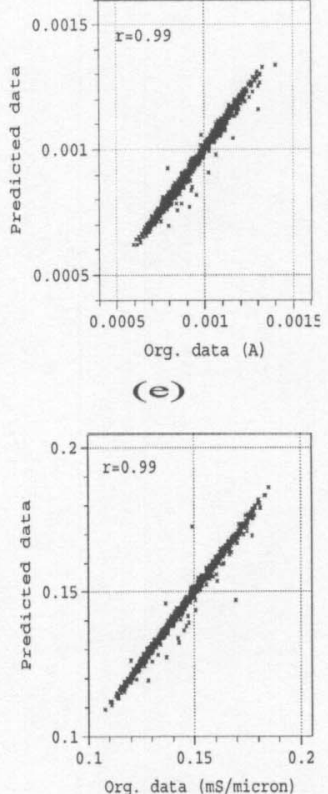

(i)

Figure 4. The correlation plots of the statistical model between the original DOE data and the model predicted data for (a) $V_{t s a t}$, (b) $V_{\text {tlin }}$, (c) $S S_{s a t}$, (d) $S S_{\text {lin }}$, (e) $I_{\text {dsatdrive, }}$, (f) $I_{\text {dsatleak }}$, (g) $I_{\text {dlindrive }}$, (h) $I_{\text {dlinleak }}$, (i) $G_{m s a t}$, and (j) $G_{m l i n}$. The correlation coefficients are also depicted for each plot

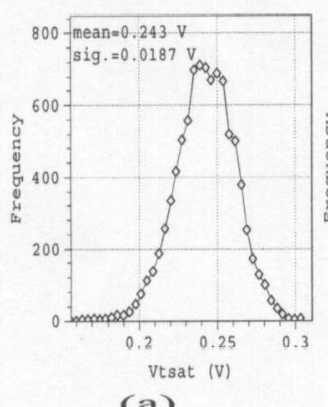

(a)

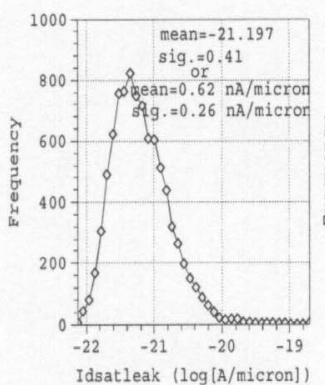

(f)

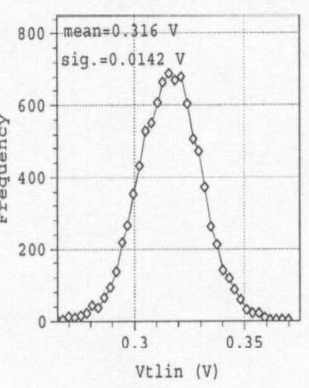

(b)

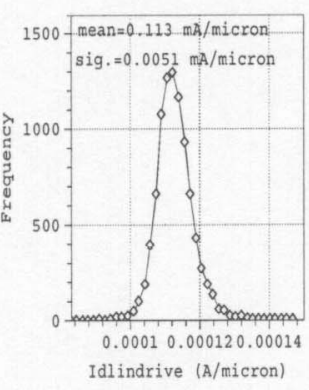

(g)
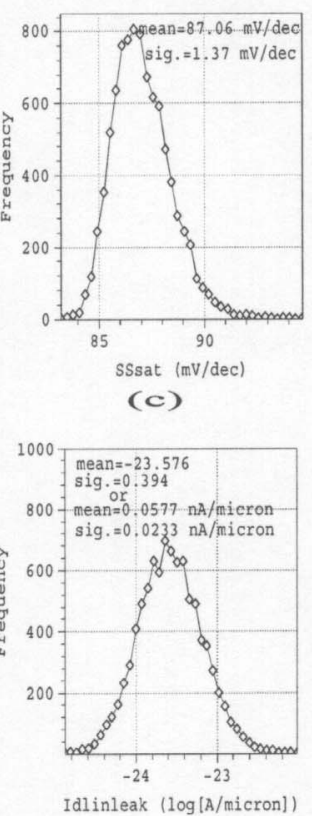

(h)
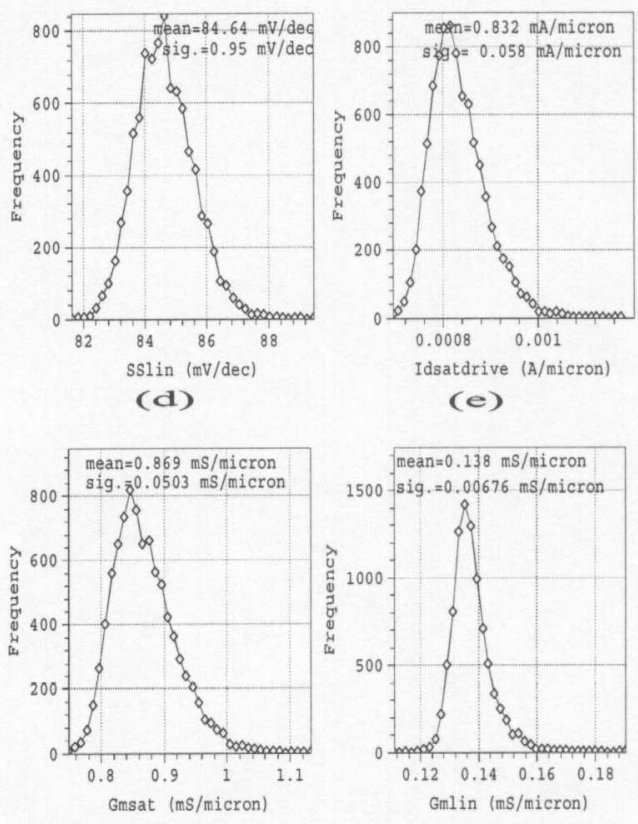

(i)

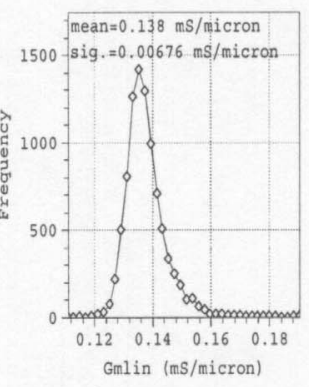

(i)

Figure 5. Statistical distributions that are obtained by the MC calculations on the models for (a) $V_{t s a t}$, (b) $V_{\text {tlin }}$, (c) $S S_{\text {sat }}$, (d) $S S_{\text {lin }}$, (e) $I_{\text {dsatdrive }}$, (f) $I_{\text {dsatleak }}$, (g) $I_{\text {dlindrive }}$, (h) $I_{\text {dlinleak }}$, (i) $G_{m s a t}$, and (j) $G_{m l i n}$. The $\mu$ and $\sigma$ for each of these quantities are also shown on each plot 
Correlation plots for these device parameters along with their correlation coefficient $r$, are depicted in Figure 4. In modeling the leakage currents, logarithmic transformation have been used, as the range of variation of these quantities with factor levels were too large, and also to improve the model fitting [8]. Therefore the model predicted data for leakage currents must be inverse transformed to interpret, with proper units. In order to study the statistical nature of $0.1 \mu \mathrm{m}$ process with respect to 10 key parameters, that are selected, we have generated 9000 normally and independently distributed pseudo-random (PR) numbers for each of these 10 key process parameters with $\mu$ equal to their nominal values and $\sigma$ equal to $3.33 \%\left(=\frac{10}{3} \%\right)$ of their nominal values. The $\sigma$ value for the temperature parameters are $3.33^{\circ} \mathrm{C}\left(=\frac{10^{\circ}}{3} \mathrm{C}\right)$. Model equation for, $V_{t}$, SS, $I_{\text {ddrive, }} I_{\text {dleak }}$ and $G_{m}$, in the saturation and linear regions are run in a Monte carlo loop by plugging in these PR numbers. The resulting distribution of these models for the corresponding device parameters are portrayed in Figure 5, along with the resulting $\mu$ and $\sigma$ values. The values of $\sigma$ for the saturation region quantities are compared with that in the literature [6] that are available for implant dose fluctuations alone. The variability that has been observed in our study is 3 to 4 times more than that, as a result of the device parameters being too sensitive to the fluctuations in the process parameters under consideration.

Finally, we have applied TMT on these models to evaluate $\mu$ and $\sigma$. The statistics that are calculated from the MC and TMT, are shown in Table 4, which match very well. In order to further validate the illustrated technique, in terms of accuracy and computational efficiency, we are currently pursuing, rigorous Monte carlo process and device simulation.

\section{Conclusions}

Characterization of $100 \mathrm{~nm}$ CMOS process has been performed on a large set of 21 process parameters, through screening experiment. Response surface study on a subset of 10 key parameters has been performed to assess the total variability (mismatch) of several NMOS device parameters. In calculating the mismatch, we have used standard techniques like, MC and TMT. The results are compared with that available in the literature for implant dose fluctuations alone. These 10 key parameters are causing nearly 3 to 4 times the fluctuations arising from implant doses. The mismatch that is arising from the process has direct impact on circuit delay fluctuations, causing switching errors in the circuit.

\section{Acknowledgment}

The authors would like to acknowledge the Department of Science and Technology, Govt. of India, for supporting this project.

\section{References}

[1]H. P. Tuinhout, A. H. Montree, J. Schmitz, and P. A. Stolk, "Effects of Gate Depletion and Boron Penetration on Matching of Deep Submicron Transistors", IEDM Technical Digest, pp. 631634, 1997.

[2]Keith A. Bowman, Xinghai Tang, John C. Eble, and James D. Meindl, "Impact of Extrinsic and Intrinsic Parameter Fluctuations on CMOS Circuit Performance", IEEE Journal of Solid-state Circuits. Vol. 35. NO. 8, pp. 1186-1193, Aug. 2000.

[3]P. A. Stolk and D. B. M. Klaassen, "The Effect of Statistical Dopant Fluctuations on MOS Device Performance", IEDM Tech. Dig., pp. 627-630, 1996.

[4]Azeez J. Bhavnagarwala, Xinghai Tang, and James D. Meindl, "The Impact of Intrinsic Device Fluctuations on CMOS SRAM Cell Stability", IEEE Journal of Solid-State Circuits, Vol. 36, NO. 4, pp. 658-665, Apr. 2001.

[5]SIA, International Technology Road map for Semiconductor, "Modeling and Simulation", 2001 Edition, available at http://public.itrs.net/Files/2001ITRS/Home.htm

[6]H. C. Srinivasaiah and Navakanta Bhat, "Monte Carlo Analysis of the Implant Dose Sensitivity in 0.1 $\mu$ m NMOSFET", Solid State Electronics, Vol. 47, No. 8, pp. 1379-1383, Aug. 2003.

[7]R.L. Plackett and J. P. Burman, "The Design of Optimum Multifactorial Experiments", Biometrika, Vol. 33, Issue 4, pp. 305-325, Jun. 1946.

[8]Douglas C. Montgomery, "Design and Analysis of Experiments", $5^{\text {th }}$ edition, John Wiley \& sons New York,

[9]George. E. P. Box and Norman R. Draper, "Empirical ModelBuilding and Response Surfaces", John Wiley and Sons Inc. International Edition, 1987. 2001.

[10]Mohammed Ismail and Terri Fiez, "Analog VLSI: Signal and Information Processing", McGraw-Hill International Editions 1994.

[11]ISE-TCAD, "ISE TCAD Release 8.0 Manual", Zurich, Switzerland, 2002.

[12]H. C. Srinivasaiah and Navakanta Bhat, "Optimization of $0.1 \mu \mathrm{m}$ NMOS Transistor using Disposable Spacer Technique", Proc. VLSI Design and Test Workshops, Bangalore, India, pp. 298-303, Aug. 2001.

[13]Yuan Taur, Douglas A. Buchanan, WeiChen, David J. Frank, Khalid E. Ismail Shih-Hsien Lo, George A. Sai-Halasz, Raman G. Vishwanathan, Hsing-Jen C. Wann, Shalom J. Wind and Hon-Sum Wong, "CMOS Scaling into Nanometer Regime", Proc. IEEE, vol. 85, No. 4, pp. 486-504, Apr. 1997.

[14]Qiuxia Xu, He Qian, Huaxiang Yin, Lin Jia, Honghao Ji, Baoqing Chen, Yajiang Zhu, Min Liu, Zhensheng Han, Huanzhang $\mathrm{Hu}$, Yulin Qiu and Dexin Wu, "The Investigation of Key Technologies for Sub-0.1 $\mu \mathrm{m}$ CMOS Devices Fabrication", IEEE Transactions on Electron Devices, Vol. 48, NO. 7, pp. 1412-1420, July 2001.

[15]H. C. Srinivasaiah and Navakanta Bhat, "Mixed-Mode Simulation Approach to Characterize the Circuit Delay Sensitivity to Implant Dose variations", IEEE Transactions on Computer Aided Design of Integrated Circuits and Systems, Vol. 22, NO. 6, pp. 742-747, June 2003.

[16]Face Centered Central Composite Design, available at, "http://www.qualitydigest.com/june01/html/doe.html/" 